\title{
Die Toxizität verschiedener Ölbekämpfungsmittel für See- und Süßwassertiere ${ }^{1}$
}

\author{
Heinrich KüHL und Hans ManN \\ Bundesforschungsanstalt für Fischerei, \\ Institut für Küsten- und Binnenfischerei, Hamburg 50
}

\begin{abstract}
The toxicity of various oil-counteracting agents for sea- and freshwater animals. Tanker accidents at sea have led to the examination of the toxic effects on organisms of 14 oil-emulsifying agents both in fresh- and sea-water. The lethal limits of the substances examined were between 0.001 and $0.1 \mathrm{ml} / 1$. For an appraisal of these values it is important to know that some substances cause different reactions in fresh-water and sea-water. Crude oil mixtures (Irak-Crude-Oil) have similar lethal limits. A layer of crude oil alone on the surface of the water is less detrimental than a mixture of crude oil and emulsifying agents. On the part of the fisheries considerable objections should be raised against the use of such agents. In cases of soilage of beaches, harbours, and industrial plants, it must be decided whether damages to the local flora and fauna can be accepted or not.
\end{abstract}

\section{EINLEITUNG}

Die steigende Zahl von Olunfällen zu Wasser und zu Lande führte dazu, natürliche und chemische Mittel zur Beseitigung der Olverunreinigungen zu benutzen, die entweder das Ol binden oder emulgieren sollen. Der Unfall des Tankschiffes "Anne Mildred Brøvig" im Februar 1966 in der Nähe von Helgoland gab Anlaß, eine Reihe von Olbekämpfungsmitteln, die dem Wasser- und Schiffahrtsamt Cuxhaven für die Olbeseitigung von der Industrie angeboten wurden, auf ihre Toxizität für verschiedene Wasserorganismen zu prüfen.

\section{METHODIK}

Für unsere Untersuchungen standen folgende Olbekämpfungsmittel zur Verfügung: Oil-Ex, Anti-Oil TS 5, Sillarit, Vecom B 24, Struktol J 520, PS 777, Gamlen, Elimax, Slix, Peroklean, Moltoclar, Hepacolith, Ascal 7-11 und Ascal ATW. Die Eignung von Emulgatoren und Ölbindemitteln für die Beseitigung von Olverschmutzungen auf Gewässern wurde bereits von Hellmann, Klein \& KNöpp (1966) bzw. STURz \& KLEIN (1964) untersucht, wobei auch die chemischen und physikalischen

${ }_{1}$ Mit Unterstïtzung der Deutschen Forschungsgemeinschaft. 
Eigenschaften dieser Materialien behandelt wurden. Wir sahen unsere Aufgabe nur darin, die Wirkung dieser Stoffe auf Fische und andere Organismen des Süß- und Seewassers zu untersuchen. Es sei aber erwähnt, daß einige von ihnen sich unterschiedlich in See- und Süßwasser verhalten, worauf später kurz eingegangen werden soll.

Im Süßwasser wurden die Olbekämpfungsmittel geprüf an: Regenbogenforellen (Salmo gairdneri) von 12 bis $15 \mathrm{~cm}$ Länge, erwachsenen Guppys (Lebistes reticulatus) und Tubificiden. Für die Untersuchungen wurde Hamburger Leitungswasser ( $\mathrm{pH} 7,4$, Härte $14,5^{\circ} \mathrm{dGH}$ ) in Vollglasaquarien benutzt. Das Wasser (Temperatur für Forellen und Tubificiden: 10 bis $15^{\circ} \mathrm{C}$, für Guppys: $23^{\circ} \mathrm{C}$ ) wurde ständig ausreichend belüftet. Die Beobachtungszeit erstreckte sich auf längstens sieben Tage.

In Brack- und Seewasser standen folgende Tiere zur Verfügung: Sarsia tubulosa, Nemopsis bachei, Pleurobrachia pileus; Larven von Spioniden und Nereiden; Copepoden (verschiedene Arten), Balanidenlarven (Nauplien und Cyprislarven), Mysideen (Mesopodopsis slabberi und Paramysis kervillei), Amphipoden (Gammariden und Coropbium); Larven von Litorina littorea; Sagitta setosa; Rotatorien (Synchaeta).

In einer weiteren Reihe wurden Versuche mit größeren Bodentieren gemacht, hauptsächlich erwachsenen Garnelen (Crangon crangon) und Strandkrabben (Carcinus maenas). Eine dritte Versuchsreihe wurde mit Jungschollen (Pleuronectes platessa) der O-Gruppe durchgeführt.

Bei den Versuchen wurde gefiltertes Brackwasser aus der Elbe bei Cuxhaven von $15 \%$ und $20 \%$ und gefiltertes Seewasser von Helgoland mit einem Salzgehalt von $32 \%$ benutzt. Die Wassertemperatur betrug 16 bis $18^{\circ} \mathrm{C}$.

Bei den Untersuchungen mit Seewasser wurde von einer Verdünnung von $1: 1000$ ausgegangen, das heißt ein Liter Seewasser bzw. Brackwasser mit einem $\mathrm{ml}$ des betreffenden Olbekämpfungsmittels intensiv gemischt. Von dieser Stammlösung wurden sogleich die weiteren Verdünnungsreihen hergestellt.

In besonderen Versuchen wurde zu den Verdünnungen Irak-Crude-Oil, das freundlicherweise von Herrn Dr. Gunkel, Helgoland, zur Verfügung gestellt wurde, tropfenweise zugegeben und kräftig umgerührt.

Im allgemeinen wurden die Untersuchungen für jede Tierart einzeln durchgeführt, gelegentlich wurde aber auch mit Mischplankton gearbeitet. Standen viele Planktonorganismen zur Verfügung, so wurde die Überlebensrate nach einer bestimmten Zeit festgestellt. Außerdem wurden aber auch Einzelversuche in kleinen Boveri-Schalen angestellt und das Verhalten der Tiere verfolgt.

\section{ERGEBNISSE}

\section{Versuche mit reinen Olbekämpfungsmitteln}

Die toxischen Wirkungen der geprüften Stoffe wurden in der Weise festgestellt, daß die Tiere ständig kontrolliert und jede Verhaltensänderung beobachtet wurde. In den meisten Fällen wurde der Tod an der Bewegungslosigkeit der Tiere festgestellt. Daneben traten aber auch andere Merkmale morphologischer Art auf, wie beispielsweise schneller Zerfall des Körpers bei Medusen (Pleurobrachia, Sarsia) oder Trübe- 
werden des sonst durchsichtigen Körpers, besonders bei Wurm- und Schneckenlarven. Verhaltensänderungen machten sich bemerkbar in Fluchtbewegungen, in Kreisschwimmen, Rückenlage, zitternden Bewegungen der Extremitäten oder Flossen (zum Beispiel bei Crangon, Carcinus und Pleuronectes) und schließlich Aufhören sämtlicher Reaktionen. Wenn man sich der Letalitätsgrenze näherte und die Todesmerkmale nicht eindeutig festzustellen waren, wurden die Tiere in frisches, unbehandeltes Wasser zurückgeführt, um zu kontrollieren, ob die Schädigungen reversibel waren.

Tabelle 1

Letalitätsgrenzen der Olbekämpfungsmittel für Süßwassertiere (Angaben in ml/1)

\begin{tabular}{|cccc|}
\hline Olbekämpfungsmittel & Regenbogenforellen & Guppy & Tubificiden \\
\hline Oil-Ex & 0,005 & 0,010 & 0,010 \\
Anti-Oil TS 5 & 0,005 & 0,050 & 0,010 \\
Sillarit & 0,005 & 0,010 & 0,005 \\
Vecom B 24 & 0,080 & 0,100 & 0,100 \\
Struktol J 502 & 0,005 & 0,005 & 0,005 \\
PS 777 & 0,010 & 0,010 & 0,010 \\
Gamlen & 0,008 & 0,020 & 0,010 \\
Elimax & 0,008 & 0,010 & 0,010 \\
Slix & 0,008 & 0,030 & 0,010 \\
Peroklean & 0,010 & 0,010 & 0,010 \\
Moltoclar & 0,010 & 0,010 & 0,010 \\
Hepacolith & 0,005 & & 0,010 \\
Ascal 7-11 & 0,005 & & 0,010 \\
Ascal ATW & & & \\
\hline
\end{tabular}

Die Ergebnisse der Versuche mit Süßwassertieren sind in Tabelle 1 aufgeführt. Aus ihr geht hervor, daß die Letalitätsgrenzen der geprüften Substanzen sich zwischen $0,005 \mathrm{ml} / 1$ und $0,100 \mathrm{ml} / 1$ bewegen. Insgesamt gesehen, ist festzustellen, daß im allgemeinen die Forellen empfindlicher sind als Guppys und Tubificiden. Für einen Großteil der geprüften Substanzen liegt die Letalitätsgrenze für Forellen bei 0,005 bis $0,008 \mathrm{ml} / 1$.

Vielfach wurde beobachtet, daß sich die Tiere anfänglich sehr unruhig verhielten, taumelten oder auf der Seite lagen, nach einiger Zeit (Stunden bis Tage) sich aber wieder erholten. Hierbei ist schwer zu beurteilen, ob die Anderung im Verhalten der Tiere allein an dem Versuchstier lag, das heißt, dieses sich an das Medium gewöhnt hatte, oder ob die Giftwirkung abgenommen hatte. Auch ist daran zu denken, daß qualitative oder quantitative Veränderungen in der Versuchslösung im Zusammenhang mit der Durchlüftung aufgetreten waren. Weiter unten wird auf diese Frage noch einmal eingegangen.

Die Ergebnisse mit den Brack- und Seewassertieren zeigt Tabelle 2. Aus ihr geht hervor, daß die Letalitätsgrenzen, im ganzen gesehen, sich zwischen 0,001 und $0,1 \mathrm{ml} / 1$ bewegen. Es sind deutlich zwei Gruppen zu unterscheiden: Auf der einen Seite stehen Sillarit und Struktol mit einer Letalitätsgrenze von etwa 0,05 bis $0,1 \mathrm{ml} / 1$, während auf der anderen Seite die Stoffe mit höherer Giftwirkung zu finden sind, bei denen die Letalitätsgrenze $z$ wischen 0,001 und $0,007 \mathrm{ml} / 1$ liegt.

Vergleicht man die Wirkung der Substanzen auf die einzelnen Tierarten, so zeigt sich, daß diejenigen Formen, die ein Außenskelett in Form von inkrustierten Chitin- 
Tabelle 2

Letalitätsgrenzen der Olbekämpfungsmittel für Seetiere (Angaben in ml/l)

\begin{tabular}{|c|c|c|c|c|c|c|c|c|c|c|}
\hline $\begin{array}{l}\text { Olbekämp- } \\
\text { fungsmittel }\end{array}$ & $\begin{array}{l}\text { Spio- } \\
\text { niden- } \\
\text { larven }\end{array}$ & $\begin{array}{l}\text { Cope- } \\
\text { poden }\end{array}$ & $\begin{array}{l}\text { Bala } \\
\text { Nau- } \\
\text { plius }\end{array}$ & $\begin{array}{l}\text { niden } \\
\text { Cypris }\end{array}$ & $\begin{array}{c}\text { Gam- } \\
\text { mari- } \\
\text { den }\end{array}$ & $\begin{array}{l}\text { Rota- } \\
\text { torien }\end{array}$ & $\begin{array}{c}\text { Schnek- } \\
\text { ken- } \\
\text { larven }\end{array}$ & $\begin{array}{c}\text { Schol- } \\
\text { len }\end{array}$ & $\begin{array}{c}\text { Crat } \\
\text { ohne } \\
\text { Ol }\end{array}$ & $\begin{array}{l}\text { gon } \\
\text { mir }\end{array}$ \\
\hline Oil- & 0,010 & & 0,010 & 0,010 & 0,010 & & \multirow[t]{6}{*}{0,010} & 0 & 0,007 & 0,009 \\
\hline Anti & & & & & & & & & 0,007 & 0,009 \\
\hline Sillarit & 0,050 & 0,050 & 0,030 & 0,050 & 0,050 & & & 0,0025 & 0,050 & 0,014 \\
\hline Vecom B 24 & 0,010 & 0,010 & 0,010 & 0,010 & 0,010 & & & 0,010 & 011 & 0,012 \\
\hline Struktol J 502 & 0,100 & & 0,100 & 0,100 & 0,100 & & & 0,010 & 0,050 & 0,012 \\
\hline PS 777 & 0,010 & 0,01 & 0,010 & 0,012 & 0,010 & 0,010 & & 0,005 & 0,012 & 0,010 \\
\hline Gamlen & 0,014 & 0,011 & 0,014 & 0,014 & & & \multirow[t]{2}{*}{0,014} & 0,001 & 0,014 & 0,011 \\
\hline Elima & 0,017 & 0,011 & 0,011 & 0,011 & 0,011 & 0,010 & & 0,010 & 0,012 & 0,011 \\
\hline Slix & 0,017 & 0,020 & 0,020 & 0,020 & & & \multirow{3}{*}{$\begin{array}{l}0,017 \\
0,010\end{array}$} & 0,0 & 0,010 & 0,006 \\
\hline Perok & 0,010 & 0,010 & 0,010 & 0,010 & 0,010 & 0,005 & & 0,0025 & 0,010 & 0,011 \\
\hline Molt & & & & & & & & 0,007 & 0,010 & 0,011 \\
\hline
\end{tabular}

panzern oder Chitinhüllen besitzen, stärkere Lösungen vertragen. Bei Fischen dagegen und auch Medusen - letztere sind allerdings in der Tabelle nicht aufgeführt - liegt die Letalitätsgrenze um eine oder zwei Potenzen höher. Besonders deutlich zeigte sich dies, wenn Mischplankton für die Untersuchung verwendet wurde. In diesem starben die Medusen sehr viel schneller als weniger empfindliche Krebs- und Wurmlarven. Auch bei den Untersuchungen an Vertretern der Süßwasserfauna kam dies zum Ausdruck, da die Tubificiden stets höhere Konzentrationen vertrugen als die Fische.

Bei einigen der untersuchten Mittel, wie zum Beispiel PS 777, Sillarit und Struktol J 502, traten bei der Mischung mit Seewasser entweder sofort oder nach einiger Zeit deutliche Flodkenbildungen auf. In diesen Flodken blieben kleinere Plankter leicht hängen und gingen dann innerhalb kurzer Zeit ein. So konnte es geschehen, daß aktive Schwimmer unter den Planktern unter den gleichen Bedingungen länger am Leben blieben als solche, die mit den Flocken absanken und dann im Bodensatz starben.

Aus den Untersuchungen, besonders bei der Herstellung größerer Verdünnungen, ging hervor, daß die toxische Wirkung einiger Olbekämpfungsmittel im Laufe der Untersuchungszeit nachließ. So konnte beispielsweise für Elimax, Moltoclar, Anti-Oil und Oil-Ex festgestellt werden, daß eine sofort angesetzte Lösung von $0,01 \mathrm{ml} / 11 \mathrm{bis}$ 3 Stunden je nach Art des Präparates tödlich wirkte, dagegen, wenn die gleiche Verdünnung sieben Tage gestanden hatte, der Tod der Testtiere (Crangon, Carcinus und Spionidenlarven) erst nach 90 bis 100 Stunden eintrat. Das entsprach bei gleicher Einwirkungsdauer einer 10fach schwächeren Lösung. Ahnliche Beobachtungen wurden oben für das Süßwasser auch schon angeführt.

Versuche mit Olbekämpfungsmitteln und Irak-Crude-Oil

In einer weiteren Versuchsreihe wurde zu den Mischungen von Olbekämpfungsmittel und Seewasser eine geringe Menge (1 bis $3 \mathrm{ml}$ auf 11 Seewasser) Irak-Crude-Oil 
gegeben und entsprechend vermischt. Diese Versuche wurden nur mit Crangon und Carcinus durchgeführt.

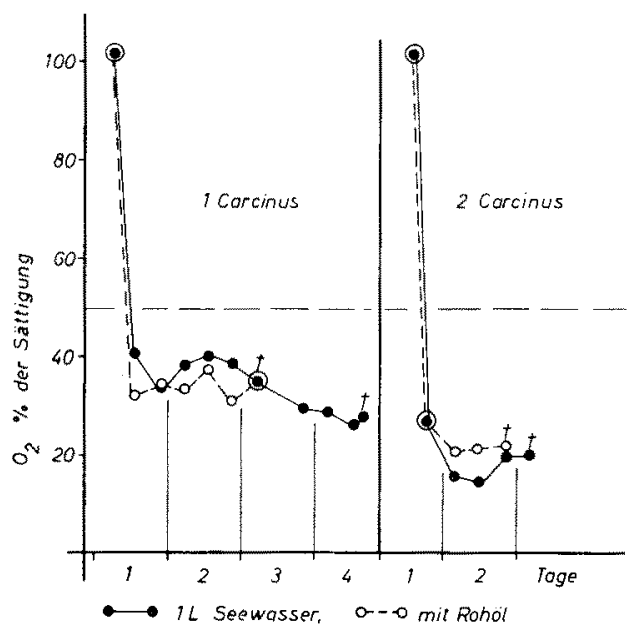

Abb. 1: Strandkrabben (Carcinus maenas) in reinem Seewasser (ausgezogene Linien) bzw. Seewasser mit Olüberschichtung (gestrichelte Linien) ohne Durchlïfung. $\uparrow$ Tod der Versuchstiere

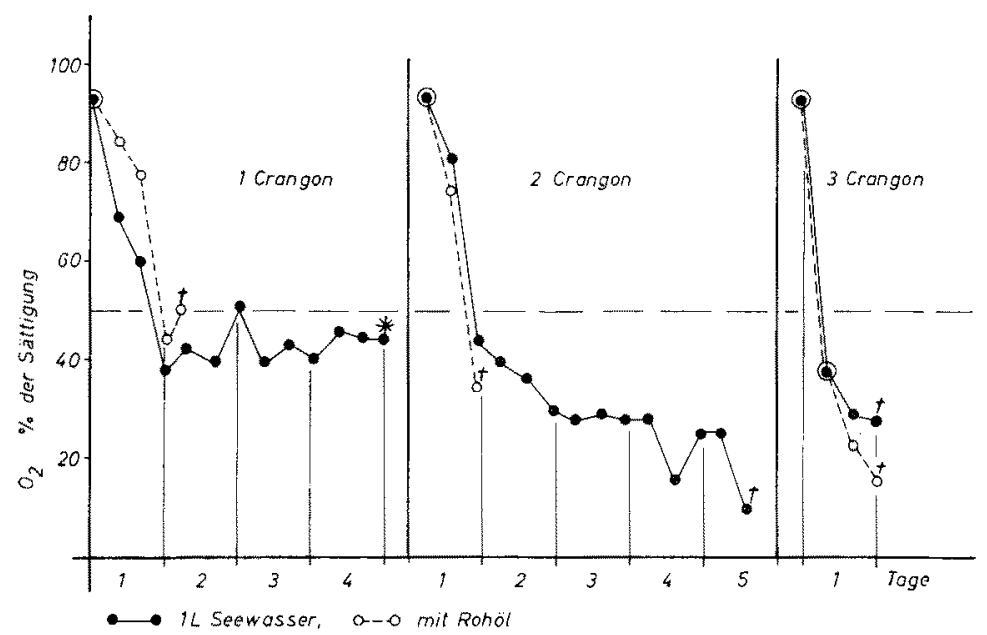

Abb. 2: Crangon in reinem Seewasser (ausgezogene Linien) bzw. Seewasser mit Olüberschichtung (gestrichelte Linien) ohne Durchlüftung. $\uparrow$ Tod der Versuchstiere. * Versuchstier lebte bis zur Beendigung des Versuds

In der Tabelle 2 sind die Letalitätsgrenzen für Crangon (Versuche ohne $\mathrm{Ol}$ und mit Oil) unter diesen Bedingungen aufgeführt. Die Gegenüberstellung zeigt, daß durch die Mischung des Olbekämpfungsmittels mit $\mathrm{O} 1$ im allgemeinen die Toxizität nicht grundlegend verändert wird. Kleine Unterschiede in der Höhe der Letalitätsgrenze 
sind zu bemerken; nur bei den Präparaten Sillarit, Struktol und Slix treten etwas größere Abweichungen auf, und zwar in der Richtung, daß die Toxizität bei einer Mischung mit Ol etwas vermindert wird. Offenbar beruht dies darauf, daß ein Teil der giftigen Inhaltsstoffe der Olbekämpfungsmittel vom $\mathrm{Ol}$ adsorbiert wird und damit nicht zur Wirkung kommt.

In zusätzlichen Versuchen wurden in Standgefäßen 1 bis 5 Exemplare von Crangon oder Carcinus ohne Durchlüftung gehalten; der Sauerstoffgehalt des Seewassers wurde laufend bestimmt. In einem Parallelversuch wurde die Wasseroberfläche durch Rohöl abgedeckt. Schließlich wurde in einer dritten Reihe die Olschicht durch eine Reihe von Olbekämpfungsmitteln „emulgiert“. In den Abbildungen 1 und 2 sind einige charakteristische Ergebnisse dargestellt. Die Kurven zeigen, daß der Sauerstoffgehalt als Folge des Sauerstoffverbrauchs durch die Atmung auf 50 bis $30 \%$ der Sättigung absinkt. Sinkt der Saverstoffgehalt unter eine bestimmte Grenze, so sterben die Tiere naturgemäß ab.

Aus dem Vergleich der Kurven ist abzulesen, daß die Tiere bei einer Olbedeckung etwas früher absterben als ohne Olbedeckung, wobei die Menge des gelösten Sauerstoffs keine Rolle spielt. Bei den Versuchen mit mehreren Tieren in einem Gefäß kommt der Unterschied nicht so deutlich zum Ausdruck, da der Sauerstoffverbrauch mehrerer Tiere höher ist und daher der Sauerstoffgehalt sehr schnell unter die letale Grenze absinkt. Offenbar treten aus dem Irak-Crude-Oil gewisse wasserlösliche Stoffe aus, die für die Krebse toxisch sind.

Gibt man nun aber zu der Olschicht tropfenweise oder mit einem Sprühgerät O1bekämpfungsmittel, so werden die Tiere sofort unruhig und gehen spätestens nach 10 Minuten ein. Der Tod tritt schon bei einer Menge des zugesetzten Präparates ein, die für eine „Emulgierung“ des Oles absolut unzureichend ist.

\section{DISKUSSION}

Die Untersuchungen an einigen Ólbekämpfungsmitteln haben deutlich gemacht, daß die geprüften Präparate gegenüber den von uns eingesetzten Testorganismen übereinstimmend eine bohe Toxizität aufweisen, obwohl die Untersuchungsmethodik, wie oben geschildert, für Süß- und Seewassertiere etwas unterschiedlich war. Die Letalitätsgrenzen bewegen sich zwischen 0,001 und $0,1 \mathrm{ml} / 1$, wobei herauszustellen ist, daß diese Werte gelten, obwohl die untersuchten Olbekämpfungsmittel offenbar von unterschiedlicher Zusammensetzung sind. Für die Beurteilung der Toxizitätswerte ist von Bedeutung, daß einige der geprïften Präparate sich in Süß- und Seewasser unterschiedlich verhalten, was mit der Natur dieser Stoffe zusammenhängt, worauf wir aber nicht näher eingehen wollen. Einige grundsätzliche Fragen zu diesem Problem sind bereits von HeLlmanN, KLEIN \& KNöpp (1966) erörtert worden. Es sei aber angeführt, daß zum Beispiel Anti-Oil TS 5, Vecom B 24, Struktol und Gamlen in Mischung mit Seewasser sich stark trübten, während dies im Süßwasser nicht der Fall war. Bei Moltoclar und Oil-Ex trat in beiden Wasserarten eine starke Trübung auf. Andererseits flockt PS 777 in Seewasser aus und bleibt in Süßwasser klar. Auf die Bedeutung der Trübung und Ausflockung wurde oben schon hingewiesen. 
Weiterhin zeigte sich, daß eine reine Olschicht auf dem Wasser keine so schädliche Wirkung ausübte wie eine Mischung von OOl mit einem der untersuchten Präparate. Letztere Beobachtung stimmt gut mit den Erfahrungen aus der Praxis überein. Bei dem Unglück mit dem Tanker "Anne Mildred Brøvig“ konnten keine Beobachtungen in dieser Richtung gesammelt werden, weil die ausgetretene Olmenge zu gering war und die deutschen Küstengebiete nicht erreicht wurden. Dagegen liegen viele Erfahrungen über den Unfall des Tankers „Torrey Canyon“ vor, aus denen hervorgeht, daß an den englischen Küsten durch die Olverschmutzung allein an den betroffenen Stellen etwa 30\% der Benthosorganismen vernichtet wurden. In Verbindung mit den Olbekämpfungsmitteln waren es jedoch $90 \%$ (NeLson-SMITH 1967). Auch dies unterstreicht, daß die bisher angewandten bzw. vorgeschlagenen Ölbekämpfungsmittel vom biologischen Standpunkt aus abzulehnen sind. Es mag dahingestellt bleiben, ob in besonderen Fällen, zum Beispiel Reinigung von Häfen, Ufer- und Industrieanlagen, man die Schädigung der Organismen in Kauf nimmt, wenn die Beseitigung der Olverschmutzung durch geeignete Mittel vordringlich ist.

\section{ZUSAMMENFASSUNG}

1. Es wurden die Letalitätsgrenzen an Süß- und Seewassertieren für folgende Olbekämpfungsmittel festgestellt: Oil-Ex, Anti-Oil TS 5, Sillarit, Vecom B 24, Struktol J 502, PS 777, Gamlen, Elimax, Slix, Peroklean, Moltoclar, Hepacolith, Ascal 7-11 und Ascal ATW.

2. Die Letalitätsgrenzen liegen $z$ wischen 0,001 und $0,100 \mathrm{ml} / 1$.

3. Einige der geprüften Olbekämpfungsmittel verhalten sich unterschiedlich in Süßund Seewasser.

4. Mischungen von Rohöl mit Olbekämpfungsmitteln haben ähnliche Letalitätsgrenzen wie die Präparate allein.

5. Bei dem Einsatz der bisher bekannten Olbekämpfungsmittel sind die Schäden an der Tierwelt weniger auf das Ol als auf die Bekämpfungsmittel zurückzuführen.

\section{ZITIERTE LITERATUR}

Hellmann, H., Klein, K. \& KNöpp, H., 1966. Untersuchungen über die Eignung von Emulgatoren für die Beseitigung von $\mathrm{Ol}$ auf Gewässern. Dt. gevässerk. Mitt. 10, 29-35; 60-70.

KüHL, H. \& MANN, H., 1967. Die Bekämpfung von Olverschmutzungen unter fischereilichen Gesichtspunkten. Schiff Hafen 19, 588-589.

Nelson-Smith, A., 1967. Oil, emulsifiers and marine life. In: Conservation and the Torrey Canyon. J. Devon Trust Nat. Cons. (Suppl.) 1967 (7), 29-33.

STURz, O. \& KLEIN, R., 1964. Erprobung von Bindemitteln zur Beseitigung von Olverunreinigungen auf Wasseroberflächen. Dt. gewässerk. Mitt. 8, 127-138. 